Article

\title{
Exploring Folate Diversity in Wild and Primitive Potatoes for Modern Crop Improvement
}

\author{
Bruce R. Robinson ${ }^{1,2}$, Vidyasagar Sathuvalli ${ }^{1,2}$, John Bamberg ${ }^{3}$ and Aymeric Goyer 1,4,* \\ 1 Hermiston Agricultural Research and Extension Center, Oregon State University, \\ Hermiston, OR 97838, USA; E-Mails: Bruce.Robinson@oregonstate.edu (B.R.R.); \\ Vidyasagar@oregonstate.edu (V.S.) \\ 2 Department of Crop and Soil Science, Oregon State University, Corvallis, OR 97330, USA \\ 3 USDA/Agricultural Research Service, Sturgeon Bay, WI 54235, USA; \\ E-Mail: john.bamberg@ars.usda.gov \\ 4 Department of Botany and Plant Pathology, Oregon State University, Corvallis, OR 97330, USA \\ * Author to whom correspondence should be addressed; E-Mail: aymeric.goyer@oregonstate.edu; \\ Tel.: +1-541-567-8321; Fax: +1-541-567-2240.
}

Academic Editors: Sean Mayes, Festo Massawe, Prakit Somta and Wai Kuan Ho

Received: 27 October 2015 / Accepted: 25 November 2015 / Published: 8 December 2015

\begin{abstract}
Malnutrition is one of the world's largest health concerns. Folate (also known as vitamin $\mathrm{B}_{9}$ ) is essential in the human diet, and without adequate folate intake, several serious health concerns, such as congenital birth defects and an increased risk of stroke and heart disease, can occur. Most people's folate intake remains sub-optimal, even in countries that have a folic acid food fortification program in place. Staple crops, such as potatoes, represent an appropriate organism for biofortification through traditional breeding based on their worldwide consumption and the fact that modern cultivars only contain about $6 \%$ of the daily recommended intake of folate. To start breeding potatoes with enhanced folate content, high folate potato material must be identified. In this study, 250 individual plants from 77 accessions and 10 Solanum species were screened for their folate content using a tri-enzyme extraction and microbial assay. There was a 10-fold range of folate concentrations among individuals. Certain individuals within the species Solanum tuberosum subsp. andigenum, Solanum vernei and Solanum boliviense have the potential to produce more than double the folate concentrations of commercial cultivars, such as Russet Burbank. Our results show that tapping into the genetic diversity of potato is a promising approach to increase the folate content of this important crop.
\end{abstract}


Keywords: vitamin B9; folate; biofortification; potato; Solanum tuberosum; andigenum; vernei

\section{Introduction}

Tetrahydrofolate (THF or vitamin B9) and its derivatives, commonly known as folates, are essential micronutrients in the human diet. Essential micronutrients refer to nutrients that are required by humans in small amounts from the diet, and if lacking, symptoms of deficiency occur. Folates are crucial for many cellular functions, including nucleic acid synthesis, the metabolism of the amino acids methionine, glycine, serine, histidine and glutamic acid and methylation reactions [1-4]. Because humans cannot synthesize folate de novo and must obtain it from their diet, adequate folate intake is critical for overall nutritional health. Chronic folate deficiency has been linked to several serious diseases, such as congenital birth defects, anemia, increased risk of stroke, certain types of cardiovascular diseases and cancers [5-7]. Low folate levels have also been linked to impaired cognitive performance and depression [8-10]. Research suggests that folic acid supplementation or adequate folate intake can improve the effectiveness of anti-depression medications $[9,10]$. Unfortunately, folate intake remains suboptimal, even in countries that have implemented industrial folic acid fortification of staple foods [11-13]. A complementary strategy to folic acid food fortification is biofortification. Biofortification is the process by which the nutritional quality of food crops is improved through biological means, such as conventional breeding (World Health Organization). It differs from food fortification, which is a manual post-harvest processing method, in that it enhances the plant's natural ability to produce increased amounts of nutrients. There are two primary approaches to biofortification, genetic engineering and traditional breeding, both of which could complement folic acid food fortification [14]. Traditional breeding has the advantage of being more accepted by the general public compared to genetically-engineered crops. Traditional breeding also offers an alternative strategy to genetic engineering strategies, which although successful for some crops, have failed in potato up to now [14]. In either case, biofortification of crops may be able to decrease folate deficiencies in at-risk populations and in less developed regions of the world, where the funding and infrastructure for industrial folic acid food fortification does not exist.

Potatoes represent an appropriate vehicle for enhanced nutrition for several reasons. Their cultivation distribution is enormous, accounting for over 300 million tonnes grown in over 150 countries in 2014 [15]. China and India are now the world's largest producers of potatoes, and their production is still increasing [16]. Half of the world's root and tuber crop are potatoes, and they are considered to be the fourth most important food crop in the world, third in terms of human consumption [15,17]. It is estimated that over one billion people worldwide consume potatoes regularly [15]. Consumption is rapidly growing in Africa and Latin America. Potatoes have the ability to produce more calories and tonnage per acre than any other crop [16]. Therefore, potatoes may be the best option to help feed a growing population in a world where food security is a serious concern.

Independent studies in several countries have reported on the contribution of potatoes to dietary folate intake. A study of a randomly-selected Finnish population found that potatoes account for $10 \%$ of the total folate intake in middle-aged men and represent one of the best sources for folate in the diet $[18,19]$. A similar study in The Netherlands found that potatoes account for $7 \%$ of the daily 
folate intake for adults and were the third greatest contributor to dietary folate intake [12]. A Greek nutritional study found that people who had an increased intake of potatoes were at a significantly decreased risk of low folate serum levels [20].

Previously, we have shown that modern potato genotypes have a relatively narrow range of folate concentrations, ranging between 400 and $1300 \mathrm{ng} \cdot \mathrm{g}^{-1}$ folate dry weight, while significantly higher folate concentrations could be found in some wild species and primitive cultivars, with concentrations two- to four-fold higher than in commercial varieties, such as Russet Burbank [21,22]. Species, such as S. boliviense, $S$. vernei and $S$. tuberosum subsp. andigenum, have accessions that could be promising sources of high folate trait [22]. In this study, the screening effort was expanded within these promising species, as well as into other wild and primitive cultivated potato species that had not been screened in order to further evaluate the natural variation of folate content in potatoes and to identify germplasm appropriate for breeding high folate trait(s) into modern cultivars.

\section{Experimental Section}

\subsection{Chemicals and Reagents}

Folate (5-formyltetrahydrofolate (5-formyl-THF)) standard was obtained from Schircks Laboratories (Jona, Switzerland). Rat plasma conjugase was obtained from Rockland Laboratories (Limerick, PA, USA) and was dialyzed before use, as described previously [23]. Difco folic acid casei medium and Lactobacilli Broth AOAC were from Becton, Dickinson, and Company (Sparks, MD, USA). All other chemicals (protease, $\alpha$-amylase) were obtained from Sigma Chemical.

\subsection{Potato Material}

Folate screening for wild and primitive cultivated species included 250 individual plants from 77 accessions and 10 species (S. stipuloideum, S. chacoense subsp. chacoense, S. candolleanum, S. acaule, S. demissum, S. microdontum, S. okadae, S. tuberosum subsp. andigenum, S. boliviense, $S$. vernei) (Table 1). The species $S$. tuberosum subsp. andigenum, S. boliviense and $S$. vernei were selected based on previous data, which showed that they could contain accessions with high folate [22]. The species $S$. stipuloideum, $S$. candolleanum, S. acaule, S. demissum, S. microdontum and S. okadae were selected because no or very few accessions within these species had been previously evaluated $[21,22]$. S. chacoense subsp. chacoense was evaluated because it is one of the most widely distributed wild potato species. Russet Burbank, a commercial variety largely grown in North America, was used as the standard [22]. Seeds of wild and primitive cultivated species were obtained from the U.S. potato gene bank (USDA Agricultural Research Service Germplasm Resource Information Network (GRIN), www.ars-grin.gov). Seeds were soaked in GA3 at $1000 \mathrm{mg} / \mathrm{L}$ overnight before planting to Metro-mix in June 2014. When plantlets reached about $8 \mathrm{~cm}$ high, they were transplanted in $8 \mathrm{~cm}$ square individual pots containing Sunshine ${ }^{\circledR}$ LA4 P. All-purpose fertilizer 20-20-20 was applied at $200 \mathrm{mg} / \mathrm{L}$ once a week until senescence. Plants were watered twice a week until senescence. Vines were killed on 31 October 2014, and tubers were harvested on 11 November. Greenhouse temperature was set at $21{ }^{\circ} \mathrm{C}$ day time and $15{ }^{\circ} \mathrm{C}$ night time. Supplemental light was provided for $14 \mathrm{~h}$ per day from a mixture of 400-Watt high pressure sodium and 1000-Watt metal halide lamps. 
Table 1. Classification, available accessions, ploidy level, accessions evaluated and their geographic origin. GRIN, Germplasm Resource Information Network.

\begin{tabular}{|c|c|c|c|c|c|c|}
\hline $\begin{array}{l}\text { Species: Spooner } \\
\text { Classification [24] }\end{array}$ & Species: Old Classification & $\begin{array}{l}\text { Number of Accessions } \\
\text { Available from GRIN }\end{array}$ & Ploidy Level & $\begin{array}{c}\text { Number of } \\
\text { Accessions Tested }\end{array}$ & PI Number Tested & Origin \\
\hline \multirow{3}{*}{ S. stipuloideum } & \multirow{3}{*}{ S. circaeifolium } & \multirow{3}{*}{14} & \multirow{3}{*}{$2 \times$} & \multirow{3}{*}{3} & 498116 & Cochabamba, Bolivia \\
\hline & & & & & 498120 & Santa Cruz, Bolivia \\
\hline & & & & & 545974 & La Paz, Bolivia \\
\hline \multirow{2}{*}{ S. chacoense } & \multirow{2}{*}{ S. chacoense subsp. chacoense } & \multirow{2}{*}{174} & \multirow{2}{*}{$2 \times$} & \multirow{2}{*}{2} & 197760 & $?$ \\
\hline & & & & & 320293 & Salta, Argentina \\
\hline \multirow{3}{*}{ S. candolleanum } & \multirow{3}{*}{ S. bukasovii } & \multirow{3}{*}{176} & \multirow{3}{*}{$2 \times$} & \multirow{3}{*}{3} & 265863 & Puno, Peru \\
\hline & & & & & 365321 & Huanuco, Peru \\
\hline & & & & & 458379 & Apurimac, Peru \\
\hline \multirow{2}{*}{ S. acaule } & \multirow{2}{*}{ S. acaule f. acaule } & \multirow{2}{*}{424} & \multirow{2}{*}{$4 \times$} & \multirow{2}{*}{3} & 175395,472661 & Argentina \\
\hline & & & & & 473481 & Huancavelica, Peru \\
\hline \multirow{3}{*}{ S. demissum } & \multirow{3}{*}{ S. demissum } & \multirow{3}{*}{164} & \multirow{3}{*}{$6 \times$} & \multirow{3}{*}{3} & 160208 & Mexico \\
\hline & & & & & 230589 & Huanuco, Peru \\
\hline & & & & & 498232 & Apurimac, Peru \\
\hline \multirow{2}{*}{ S. microdontum } & \multirow{2}{*}{ S. microdontum subsp. microdontum } & \multirow{2}{*}{116} & \multirow{2}{*}{$2 \times$} & \multirow{2}{*}{2} & 458355 & Jujuy, Argentina \\
\hline & & & & & 498123 & Chuquisaca, Bolivia \\
\hline \multirow{3}{*}{ S. okadae } & S. venturii & \multirow{3}{*}{16} & $2 \times$ & 1 & 458368 & Salta, Argentina \\
\hline & \multirow{2}{*}{ S. okadae } & & 0 & 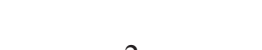 & 498130 & Cochabamba, Bolivia \\
\hline & & & $2 \times$ & 2 & 320327 & Salta, Argentina \\
\hline & $S$ r & & $2 \times$ & 2 & 195204 & Cuzco, Peru \\
\hline & S. stenotomum subsp. stenotomит & & $2 \times$ & 2 & 283141 & Colombia \\
\hline & S nhureig subs nhureig & & $2 \times$ & 3 & 320355,320377 & Narino, Colombia \\
\hline S. tuberosum subsp. andigenum & S. prareja suosp. praveja & 1006 & $2 \lambda$ & 3 & 225710 & Cauca, Colombia \\
\hline & & & & & 546023 & Potosi, Bolivia \\
\hline & S. tuberosum subsp. andigenum & & $4 \times$ & 3 & 607886 & Cuzco, Peru \\
\hline & & & & & 281034 & Mexico \\
\hline
\end{tabular}


Table 1. Cont.

\begin{tabular}{|c|c|c|c|c|c|c|}
\hline $\begin{array}{l}\text { Species: Spooner } \\
\text { Classification [24] }\end{array}$ & $\begin{array}{l}\text { Species: Old } \\
\text { Classification }\end{array}$ & $\begin{array}{l}\text { Number of Accessions } \\
\text { Available from GRIN }\end{array}$ & $\begin{array}{r}\text { Ploidy } \\
\text { Level }\end{array}$ & $\begin{array}{c}\text { Number of } \\
\text { Accessions Tested }\end{array}$ & PI Number Tested & Origin \\
\hline \multirow{7}{*}{ S. boliviense } & \multirow{7}{*}{ S. megistacrolobum } & \multirow{7}{*}{222} & \multirow{7}{*}{$2 \times$} & \multirow{7}{*}{26} & 283082 & Bolivia \\
\hline & & & & & 283133 & Ecuador \\
\hline & & & & & $275149,435077,500029,500030$ & Salta, Argentina \\
\hline & & & & & $\begin{array}{l}458347,458348,473110,473112,473113,473124,473129, \\
473130,473138,473141,473144,473149,473160,558094\end{array}$ & Jujuy, Argentina \\
\hline & & & & & 545899,568986 & Tarija, Bolivia \\
\hline & & & & & 597689 & Oruro, Bolivia \\
\hline & & & & & $597705,597706,597736$ & Potosi, Bolivia \\
\hline \multirow{7}{*}{ S. vernei } & \multirow{4}{*}{ S. vernei subsp. vernei } & \multirow{6}{*}{36} & \multirow{4}{*}{$2 \times$} & \multirow{4}{*}{18} & 320332 & Catamarca, Argentina \\
\hline & & & & & $230468,458373,473308$ & Tucuman, Argentina \\
\hline & & & & & $\begin{array}{c}458374,473306,473310,473311,500045,500062,500063 \\
500065,558147,558148\end{array}$ & Salta, Argentina \\
\hline & & & & & $500067,500069,558149,558150$ & Jujuy, Argentina \\
\hline & \multirow{2}{*}{ S. vernei subsp. ballsii } & & \multirow{2}{*}{$2 \times$} & \multirow{2}{*}{5} & 458369,473303 & Jujuy, Argentina \\
\hline & & & & & $458370,458371,458372$ & Salta, Argentina \\
\hline & S. vernei & & $2 \times$ & 1 & 500066 & Jujuy, Argentina \\
\hline
\end{tabular}


One to 4 individual plants per accession, with a minimum of 3 in most instances, were grown. One individual plant is a plant from one botanical seed. A representative set of tubers from one individual plant was pooled and processed together as follows. Tubers were left with skin intact, washed with cold water in a strainer, weighed and then flash-frozen with liquid nitrogen before storage at $-80{ }^{\circ} \mathrm{C}$. A few tubers from each genotype were stored at $4{ }^{\circ} \mathrm{C}$ as back-up for re-planting. Frozen samples were then lyophilized in a freeze-dryer (VirTis Benchtop K) (vacuum pressure $<100$ mTorr) for two to three days. Dried samples were weighed, and the initial moisture content was calculated by the weight difference before and after freeze-drying potato samples [22]. Removal of water from tuber samples allows for a more consistent comparison of vitamin content among samples, because moisture content varies greatly in these materials (68\% to $82 \%$ ). Samples (i.e., one sample is made of several tubers from one individual plant) were then ground to a fine powder with a Waring blender and transferred to scintillation vials for long-term storage at $-80^{\circ} \mathrm{C}$.

\subsection{Folate Analysis}

Folates were extracted by using a tri-enzyme extraction method, as previously published [21,22]. Potato samples $(100 \mathrm{mg})$ were homogenized in $15-\mathrm{mL}$ Falcon tubes containing $10 \mathrm{~mL}$ of extraction buffer consisting of $50 \mathrm{mM}$ HEPES/50 mM CHES, $\mathrm{pH} 7.85,2 \%(w / v)$ sodium ascorbate and $10 \mathrm{mM}$ $\beta$-mercaptoethanol and deoxygenated by flushing with nitrogen. Once homogenized, samples were boiled for $10 \mathrm{~min}$ and cooled immediately on ice in a covered cooler. The homogenate was then treated with protease ( $\geq 14$ units) and incubated for $2 \mathrm{~h}$ at $37{ }^{\circ} \mathrm{C}$, boiled again for $5 \mathrm{~min}$ and cooled immediately in a covered cooler of ice. The samples were then treated with $\alpha$-amylase ( $\geq 800$ units) and rat plasma conjugase in large excess $(0.5 \mathrm{~mL} / \mathrm{sample})$, incubated for $3 \mathrm{~h}$ at $37{ }^{\circ} \mathrm{C}$, boiled again for $5 \mathrm{~min}$ and cooled immediately in a covered cooler of ice. After centrifugation at $3000 \mathrm{~g}$ for $10 \mathrm{~min}$, the supernatant was transferred to a new tube. The residue was re-suspended and homogenized in $5 \mathrm{~mL}$ of extraction buffer, re-centrifuged for $10 \mathrm{~min}$, and the supernatant was recovered. Supernatants were then combined and the samples' volume adjusted to $20 \mathrm{~mL}$ with extraction buffer. Aliquots of each sample were transferred to $1.5-\mathrm{mL}$ microcentrifuge tubes, flushed with nitrogen and stored at $-80^{\circ} \mathrm{C}$ until analysis by the microbiological assay. Controls containing all reagents, but potato samples, were used to determine the amount of any residual folates in the reagents. There were no detectable folates in any of the reagents used.

Folate concentrations were measured by microbiological assay using Lactobacillus rhamnosus. L. rhamnosus (ATCC 7469) cultures were obtained from the American Type Culture Collection (Manassas, VA, USA). Glycerol cryoprotected cells of L. rhamnosus were prepared as described previously [25]. Assays were performed in 96-well plates (Falcon microtiter plates). Wells contained growth medium supplemented with folate standards or potato extracts, each plated in triplicate. Bacterial growth was measured at $630 \mathrm{~nm}$ after $18 \mathrm{~h}, 21 \mathrm{~h}$ and $24 \mathrm{~h}$ of incubation at $37^{\circ} \mathrm{C}$. The $24-\mathrm{h}$ reading was usually used for analysis unless saturation was reached, in which case, the 21-h reading was used. All measurements were made with a BioTek Instrument EL 311 SX microplate auto-reader (BioTekInstrument, Winooski, VT, USA), analyzed with the KCJr EIA application software (BioTekInstrument, Winooski, VT, USA) and compiled in Microsoft Excel. Final results were calculated by reference to a standard curve using 5-formyl-THF and expressed as nanograms of folate per gram of dry sample. 
A large batch of dried potato powder was prepared from tubers of Solanum pinnatisectum PI 275233 and was used as the reference material. Each batch of extractions contained 18 samples plus the reference material. Values obtained for samples were normalized to values obtained for the reference material. The average folate concentration of the reference material across all of the extractions was $1105 \pm 76 \mathrm{ng} \cdot \mathrm{g}^{-1} \mathrm{DW}$. All calculations were performed with standard function settings in Microsoft Excel.

\subsection{Statistical Analysis}

One-way analysis of variance (ANOVA) was performed to compare normalized mean values of folate content in all species. The only species that was significantly different from all other species at a $p$-value $\leq 0.001$ was $S$. vernei. All statistical analysis was performed with $\mathrm{R}$ in $\mathrm{R}$-Studio with the "stats" package linear regression and ANOVA functions.

\section{Results}

Overall, folate concentrations ranged from $221 \pm 19$ to $2336 \pm 285 \mathrm{ng} \cdot \mathrm{g}^{-1}$ dry weight (Table 2), representing a 10.5-fold difference between the lowest and highest folate concentration. The majority of individuals ( $55 \%$ of all individuals tested) had folate concentrations between 500 and $1000 \mathrm{ng} \cdot \mathrm{g}^{-1}$ dry weight, including the modern variety Russet Burbank (Figure 1).

Table 2. Folate concentration (mean $\pm \mathrm{SE}$ ) in $\mathrm{ng} \cdot \mathrm{g}^{-1}$ dry matter per accession. In bold are individuals with folate concentrations higher than $1500 \mathrm{ng} \cdot \mathrm{g}^{-1}$ dry weight.

\begin{tabular}{|c|c|c|c|c|c|}
\hline $\begin{array}{c}\text { Plant Introduction } \\
\text { Number }\end{array}$ & Species & $\begin{array}{c}\text { Number of } \\
\text { Individuals Tested }\end{array}$ & $\begin{array}{c}\text { Individual } \\
\text { Measurements }\end{array}$ & Mean \pm SE & $\% \mathrm{DM}$ \\
\hline R. Burbank & $\begin{array}{c}\text { S. tuberosum } \\
\text { subsp. tuberosum }\end{array}$ & 3 & $1276-915-929$ & $1040 \pm 118$ & 26 \\
\hline 498116 & S. stipuloideum & 3 & $907-1115-1118$ & $1046 \pm 57$ & 25 \\
\hline 498120 & S. stipuloideum & 3 & $304-553-532$ & $463 \pm 65$ & 25 \\
\hline 545974 & S. stipuloideum & 4 & $415-824-1119-882$ & $810 \pm 127$ & 23 \\
\hline 197760 & S. chacoense & 3 & $591-653-653$ & $632 \pm 17$ & 36 \\
\hline 320293 & S. chacoense & 4 & $478-240-1198-408$ & $581 \pm 183$ & 37 \\
\hline 265863 & S. candolleanum & 3 & $1367-508-481$ & $786 \pm 206$ & 25 \\
\hline 365321 & S. candolleanum & 1 & 918 & $918 \pm$ n.d. & 19 \\
\hline 458379 & S. candolleanum & 1 & 1023 & $1023 \pm$ n.d. & 19 \\
\hline 175395 & S. acaule & 4 & $461-562-562-1017$ & $651 \pm 108$ & 22 \\
\hline 472661 & S. acaule & 4 & $480-490-517-1014$ & $625 \pm 113$ & 23 \\
\hline 473481 & S. acaule & 2 & $632-757$ & $695 \pm 44$ & 25 \\
\hline 160208 & S. demissum & 4 & $749-455-487-556$ & $562 \pm 57$ & 23 \\
\hline 230589 & S. demissum & 2 & $410-631$ & $520 \pm 78$ & 22 \\
\hline 498232 & S. demissum & 4 & $760-737-669-455$ & $655 \pm 60$ & 30 \\
\hline 458355 & S. microdontum & 3 & $703-694-650$ & $682 \pm 13$ & 32 \\
\hline 498123 & S. microdontum & 2 & $913-767$ & $840 \pm 51$ & 36 \\
\hline 320327 & S. okadae & 3 & $876-548-629$ & $684 \pm 80$ & 35 \\
\hline 458368 & S. okadae & 3 & $611-1317-991$ & $973 \pm 167$ & 34 \\
\hline
\end{tabular}


Table 2. Cont.

\begin{tabular}{|c|c|c|c|c|c|}
\hline $\begin{array}{c}\text { Plant Introduction } \\
\text { Number } \\
\end{array}$ & Species & $\begin{array}{c}\text { Number of } \\
\text { Individuals Tested }\end{array}$ & $\begin{array}{c}\text { Individual } \\
\text { Measurements }\end{array}$ & Mean $\pm \mathbf{S E}$ & $\% \mathrm{DM}$ \\
\hline 498130 & S. okadae & 3 & $723-660-806$ & $730 \pm 34$ & 38 \\
\hline 195204 & $\begin{array}{l}\text { S. tuberosum subsp. } \\
\text { andigenum }\end{array}$ & 4 & 410-836-499-794 & $635 \pm 92$ & 24 \\
\hline 225710 & $\begin{array}{l}\text { S. tuberosum subsp. } \\
\text { andigenum }\end{array}$ & 1 & 2337 & $2337 \pm$ n.d. & 22 \\
\hline 281034 & $\begin{array}{l}\text { S. tuberosum subsp. } \\
\text { andigenum }\end{array}$ & 4 & $565-1030-1126-506$ & $807 \pm 137$ & 18 \\
\hline 283141 & $\begin{array}{l}\text { S. tuberosum subsp. } \\
\text { andigenum }\end{array}$ & 3 & $468-457-711$ & $545 \pm 68$ & 18 \\
\hline 320355 & $\begin{array}{l}\text { S. tuberosum subsp. } \\
\text { andigenum }\end{array}$ & 2 & $853-1400$ & $1126 \pm 193$ & 28 \\
\hline 320377 & $\begin{array}{c}\text { S. tuberosum subsp. } \\
\text { andigenum }\end{array}$ & 2 & 2198-1038 & $1618 \pm 410$ & 17 \\
\hline 546023 & $\begin{array}{c}\text { S. tuberosum subsp. } \\
\text { andigenum }\end{array}$ & 4 & $985-700-333-626$ & $661 \pm 116$ & 21 \\
\hline 607886 & $\begin{array}{c}\text { S. tuberosum subsp. } \\
\text { andigenum }\end{array}$ & 4 & $404-553-622-361$ & $485 \pm 53$ & 21 \\
\hline 275149 & S. boliviense & 4 & 566-561-602-515 & $561 \pm 15$ & 24 \\
\hline 283082 & S. boliviense & 1 & 934 & $934 \pm$ n.d. & 23 \\
\hline 283133 & S. boliviense & 4 & $891-1102-1097-351$ & $860 \pm 153$ & 27 \\
\hline 435077 & S. boliviense & 3 & $421-652-630$ & $568 \pm 60$ & 26 \\
\hline 458347 & S. boliviense & 3 & $779-1393-525$ & $899 \pm 210$ & 21 \\
\hline 458348 & S. boliviense & 4 & 585-759-666-679 & $672 \pm 31$ & 23 \\
\hline 473110 & S. boliviense & 4 & $362-456-651-611$ & $520 \pm 58$ & 18 \\
\hline 473112 & S. boliviense & 4 & $517-897-450-688$ & $638 \pm 87$ & 20 \\
\hline 473113 & S. boliviense & 1 & 869 & $869 \pm$ n.d. & 20 \\
\hline 473124 & S. boliviense & 4 & $630-547-456-610$ & $561 \pm 34$ & 20 \\
\hline 473129 & S. boliviense & 4 & $816-997-722-584$ & $780 \pm 75$ & 21 \\
\hline 473130 & S. boliviense & 4 & $411-526-512-751$ & $550 \pm 62$ & 24 \\
\hline 473138 & S. boliviense & 4 & $1265-787-512-745$ & $827 \pm 137$ & 21 \\
\hline 473141 & S. boliviense & 4 & $460-524-630-385$ & $500 \pm 45$ & 23 \\
\hline 473144 & S. boliviense & 4 & $473-355-222-449$ & $375 \pm 49$ & 22 \\
\hline 473149 & S. boliviense & 4 & $628-523-826-780$ & $689 \pm 60$ & 24 \\
\hline 473160 & S. boliviense & 4 & $557-352-706-347$ & $491 \pm 75$ & 22 \\
\hline 500029 & S. boliviense & 4 & 684-461-610-622 & $594 \pm 41$ & 26 \\
\hline 500030 & S. boliviense & 4 & $541-494-575-671$ & $634 \pm 32$ & 24 \\
\hline 545899 & S. boliviense & 4 & $647-426-1033-780$ & $721 \pm 110$ & 22 \\
\hline 558094 & S. boliviense & 3 & $350-473-542$ & $455 \pm 46$ & 21 \\
\hline 568986 & S. boliviense & 2 & $684-888$ & $786 \pm 72$ & 19 \\
\hline 597689 & S. boliviense & 4 & $570-1099-723-822$ & $804 \pm 96$ & 22 \\
\hline 597705 & S. boliviense & 4 & $332-749-366-707$ & $539 \pm 95$ & 20 \\
\hline 597706 & S. boliviense & 4 & $484-567-551-623$ & $556 \pm 25$ & 23 \\
\hline
\end{tabular}


Table 2. Cont.

\begin{tabular}{|c|c|c|c|c|c|}
\hline $\begin{array}{c}\text { Plant Introduction } \\
\text { Number }\end{array}$ & Species & $\begin{array}{c}\text { Number of } \\
\text { Individuals Tested }\end{array}$ & $\begin{array}{c}\text { Individual } \\
\text { Measurements }\end{array}$ & Mean \pm SE & $\%$ DM \\
\hline 597736 & S. boliviense & 4 & 713-1947-539-777 & $994 \pm 279$ & 33 \\
\hline 230468 & S. vernei & 4 & 1377-1072-1416-1911 & $1444 \pm 150$ & 24 \\
\hline 320332 & S. vernei & 4 & 1137-846-1985-1105 & $1268 \pm 215$ & 28 \\
\hline 458369 & S. vernei & 2 & $1197-1002$ & $1099 \pm 69$ & 22 \\
\hline 458370 & S. vernei & 4 & $1207-1062-1073-817$ & $1040 \pm 70$ & 20 \\
\hline 458371 & S. vernei & 4 & 1940-786-881-1601 & $1302 \pm 242$ & 22 \\
\hline 458372 & S. vernei & 4 & 1450-1801-1023-1110 & $1346 \pm 154$ & 23 \\
\hline 458373 & S. vernei & 2 & $1316-1145$ & $1230 \pm 60$ & 26 \\
\hline 458374 & S. vernei & 4 & 891-838-851-908 & $872 \pm 14$ & 25 \\
\hline 473303 & S. vernei & 3 & 1623-1099-774 & $1165 \pm 202$ & 21 \\
\hline 473306 & S. vernei & 3 & 1968-1307-1703 & $1659 \pm 157$ & 18 \\
\hline 473308 & S. vernei & 1 & 1117 & $1117 \pm$ n.d. & 25 \\
\hline 473310 & S. vernei & 3 & $649-973-1058$ & $893 \pm 102$ & 21 \\
\hline 473311 & S. vernei & 3 & 1589-1309-1122 & $1340 \pm 111$ & 23 \\
\hline 500045 & S. vernei & 2 & $1361-826$ & $1093 \pm 189$ & 26 \\
\hline 500062 & S. vernei & 2 & $1287-818$ & $1053 \pm 166$ & 25 \\
\hline 500063 & S. vernei & 4 & $469-1282-776-725$ & $813 \pm 147$ & 22 \\
\hline 500065 & S. vernei & 3 & $829-1105-835$ & $923 \pm 74$ & 25 \\
\hline 500066 & S. vernei & 3 & $1178-1722-1372$ & $1424 \pm 112$ & 20 \\
\hline 500067 & S. vernei & 4 & $1219-1370-961-1035$ & $1146 \pm 280$ & 22 \\
\hline 500069 & S. vernei & 3 & $970-948-1294$ & $1070 \pm 91$ & 24 \\
\hline 558147 & S. vernei & 2 & $1117-1150$ & $1133 \pm 12$ & 23 \\
\hline 558148 & S. vernei & 4 & 853-974-1312-959 & $1024 \pm 86$ & 26 \\
\hline 558149 & S. vernei & 4 & 1268-2211-1688-1355 & $1630 \pm 185$ & 24 \\
\hline 558150 & S. vernei & 2 & $909-\mathbf{1 6 2 0}$ & $1264 \pm 252$ & 22 \\
\hline
\end{tabular}

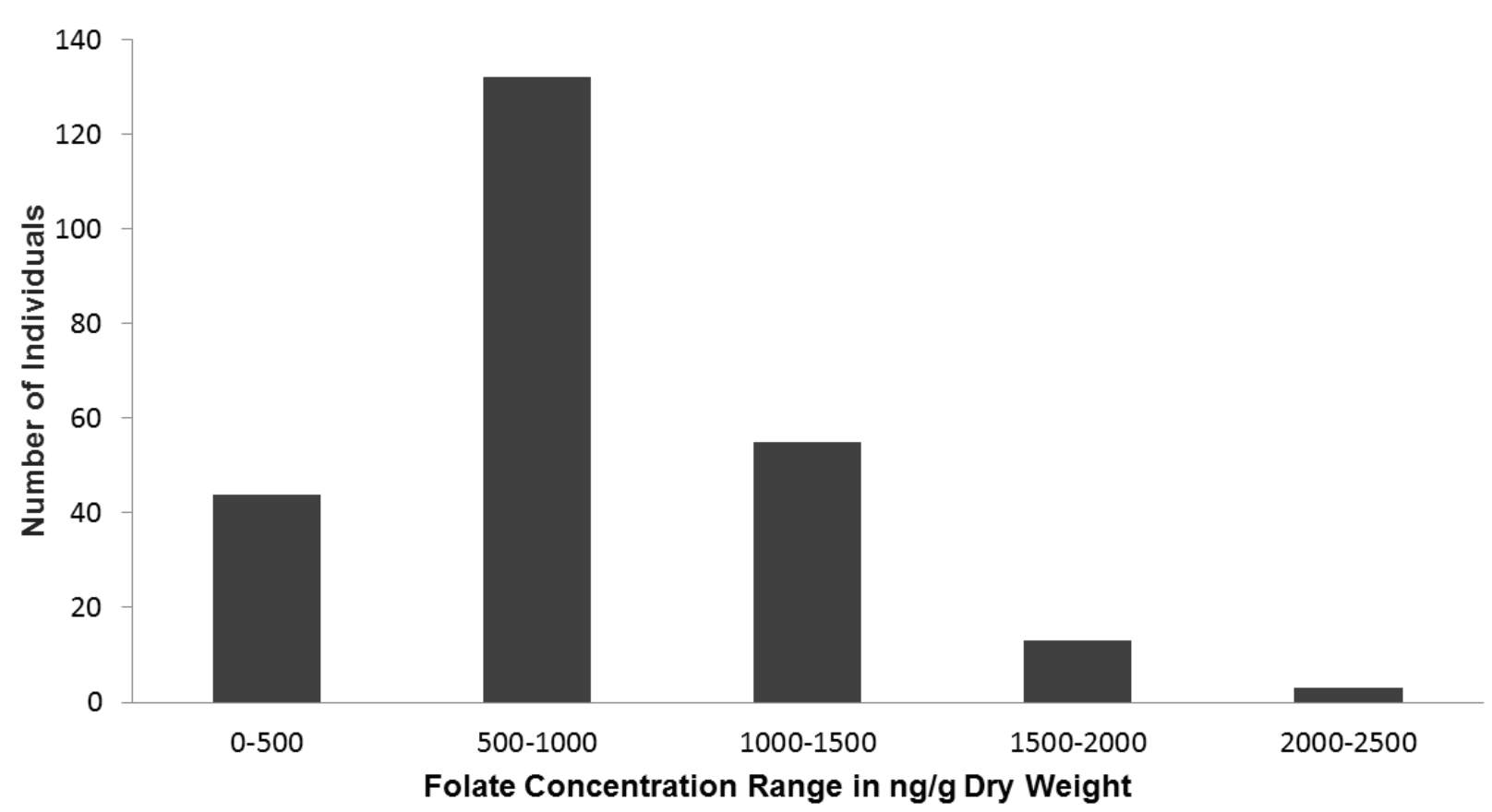

Figure 1. Histogram of number of individuals within folate concentration brackets. 
About $40 \%$ of individuals had folate concentrations below 500 or between 1000 and $1500 \mathrm{ng} \cdot \mathrm{g}^{-1}$ dry weight. The remaining $10 \%$ had folate concentrations above $1500 \mathrm{ng} \cdot \mathrm{g}^{-1}$ dry weight, with thirteen individuals between 1500 and $2000 \mathrm{ng} \cdot \mathrm{g}^{-1}$ dry weight and three above $2000 \mathrm{ng} \cdot \mathrm{g}^{-1}$ dry weight (Figure 1). In most cases, a minimum of three individual plants per accession were evaluated for folate (Table 2).

Each accession showed different levels of variability between individuals, with some accessions displaying a low level of variability (e.g., PI 197760), while other accessions had individuals with up to a five-fold folate concentration range (e.g., PI 320293). Two individuals with folate concentrations above $2000 \mathrm{ng} \cdot \mathrm{g}^{-1}$ dry weight were from the accessions PI 225710 and PI 320377, both from the species S. tuberosum subsp. andigenum. Overall, about $25 \%$ of all individuals from the species $S$. tuberosum subsp. andigenum had folate concentrations above $1000 \mathrm{ng} \cdot \mathrm{g}^{-1}$ dry weight (Table 2 and Figure 2). For S. boliviense, one individual within the accession PI 597736 contained folate concentrations of $1947 \mathrm{ng} \cdot \mathrm{g}^{-1}$ dry weight. Only $7.5 \%$ of all individuals from the species S. boliviense had folate concentrations above $1000 \mathrm{ng} \cdot \mathrm{g}^{-1}$ dry weight (Table 2 and Figure 2). For S. vernei, one individual from the accession PI 558149 had folate concentrations above $2000 \mathrm{ng} \cdot \mathrm{g}^{-1}$ dry weight, and seven individuals from six different accessions (PI 320332, PI 458371, PI 458372, PI 473306, PI 500066 and PI 558149) contained folate concentrations greater than $1700 \mathrm{ng} \cdot \mathrm{g}^{-1}$ dry weight. Over $66 \%$ of all individuals from this species had folate concentrations above $1000 \mathrm{ng} \cdot \mathrm{g}^{-1}$ dry weight (Table 2 and Figure 2). Amongst the species S. stipuloideum, S. candolleanum, S. acaule, S. demissum, S. microdontum, S. okadae and $S$. chacoense subsp. chacoense, no individual had folate concentrations above $1500 \mathrm{ng} \cdot \mathrm{g}^{-1}$ dry weight (Table 2 and Figure 2). S. demissum had the lowest maximum folate concentration (760 $\mathrm{ng} \cdot \mathrm{g}^{-1}$ dry weight), while S. candolleanum had the highest (1367 $\mathrm{ng} \cdot \mathrm{g}^{-1}$ dry weight) (Figure 2).

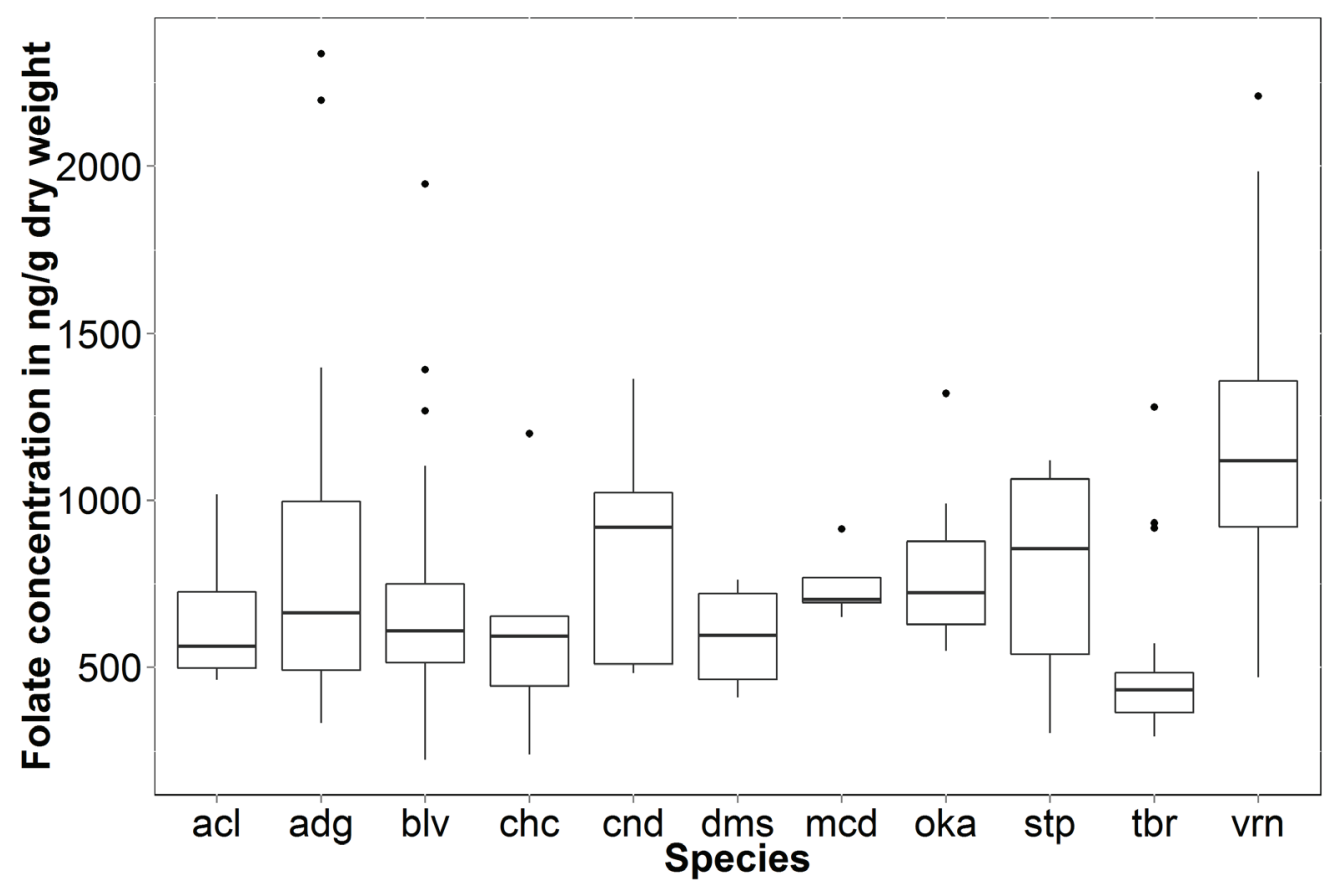

Figure 2. Box and whisker plot of folate concentrations by species. acl, S. acaule; adg, S. tuberosum subsp. andigenum; blv, S. boliviense; chc, S. chacoense subsp. chacoense; cnd, S. candolleanum; dms, S. demissum; mcd, S. microdontum; oka, S. okadae; stp, S. stipuloideum; tbr, S. tuberosum; vrn, $S$. vernei. For $S$. tuberosum, data for two varieties, Russet Burbank and Yukon Gold, each in three biological replicates, were used. 
As previously demonstrated [21,26], the peel contains a substantially higher amount of folates than the potato flesh. Because tubers from wild and primitive species are usually small, the relatively higher contribution of the peel could be responsible for at least part of the high folate concentrations observed. However, when the tuber length of five to six representative tubers for each individual tested in this study was plotted against folate concentrations, the coefficient of correlation $r$ was 0.03 (Figure A1). These results indicate that tubers of a similar size can have very different folate content and show that a tuber with a relatively higher amount of peel does not necessarily contain a higher amount of folates.

\section{Discussion}

This study shows that there is an enormous amount of genetic diversity within the germplasm that was evaluated for folate content. It also shows that $S$. tuberosum subsp. andigenum, S. boliviense and $S$. vernei all contain individuals that have the ability to produce and accumulate significantly higher concentrations of folate (over two-fold) in their tubers than a modern commercial variety, such as Russet Burbank. These individuals are promising materials for breeding potato with high folate content. S. tuberosum subsp. andigenum, S. vernei and S. boliviense were selected for evaluation based on a previous study with fewer individuals that showed that high folate concentrations could be found within these species [22]. Of particular interest is the accession PI 225710 from the species S. tuberosum subsp. andigenum from which we found the highest folate concentrations $\left(>2000 \mathrm{ng} \cdot \mathrm{g}^{-1}\right.$ dry weight). Another individual (clone named RN018.03) from the same accession that contained folate concentrations above $2000 \mathrm{ng} \cdot \mathrm{g}^{-1}$ dry weight had previously been identified [22]. Therefore, this accession may be a good source of high folate individuals. However, more individuals will need to be evaluated to confirm this hypothesis. One individual from the accession PI 320377 from the species S. tuberosum subsp. andigenum also had folate concentrations above $2000 \mathrm{ng} \cdot \mathrm{g}^{-1}$ dry weight. Other individuals from this accession were previously reported in the low to mid-range folate levels [22]. The highest folate concentration found within the species $S$. boliviense was in an individual from the accession PI 597736 (1947 $\mathrm{ng} \cdot \mathrm{g}^{-1}$ dry weight). This accession had previously provided individuals with average folate concentrations above $3000 \mathrm{ng} \cdot \mathrm{g}^{-1}$ dry weight [22] and may also be a good source of high folate individuals. It should be noted that the concentration of $3000 \mathrm{ng} \cdot \mathrm{g}^{-1}$ dry weight was found in tubers that were stored at a cold temperature for six months. We have found that cold storage could significantly increase folate concentrations [21]. Re-evaluation of one of these individuals has shown more modest folate concentrations (1500 to $2000 \mathrm{ng} \cdot \mathrm{g}^{-1}$ dry weight) in freshly-harvested tubers. Five individuals from the species $S$. vernei had folate concentrations above $1900 \mathrm{ng} \cdot \mathrm{g}^{-1}$ dry weight, including one individual from the accession PI 230468. We had previously found individuals from this accession with average folate concentrations above $1500 \mathrm{ng} \cdot \mathrm{g}^{-1}$ dry weight [22]. The accession PI 558149 had one individual with folate concentrations above $2200 \mathrm{ng} \cdot \mathrm{g}^{-1}$ dry weight and an average folate concentration for four individuals higher than $1600 \mathrm{ng} \cdot \mathrm{g}^{-1}$ dry weight. Therefore, these accessions may be other good sources of high folate individuals. The species $S$. vernei also had a large number of individuals with folate concentrations above $1000 \mathrm{ng} \cdot \mathrm{g}^{-1}$ dry weight (49 out of 74 individuals). By comparison, $S$. boliviense only had seven out of 93 individuals with folate concentrations above $1000 \mathrm{ng} \cdot \mathrm{g}^{-1}$ dry weight. $S$. vernei may therefore be a good species to further evaluate. No high folate $\left(>1500 \mathrm{ng} \cdot \mathrm{g}^{-1}\right.$ dry weight) individuals were identified in the other species evaluated in this study 
(S. stipuloideum, S. candolleanum, S. acaule, S. demissum, S. microdontum, S. okadae and S. chacoense subsp. chacoense). Although one cannot preclude that high folate individuals could still be identified by extending the screening, our results also indicate that these species may not be the best genetic pool to screen for high folate individuals.

Although our data indicate that some accessions and some species may be better sources of high folate individuals than others, our results also illustrate the high degree of variability within accessions and species. Thus, until a larger number of individuals are being evaluated within each accession and species, it is currently difficult to pinpoint, with high confidence, a specific accession and/or species for high folate content. It should be emphasized that screening wild or primitive potato species for folate is very tedious. First, folate analyses are very time consuming. Second, tubers from wild and primitive species are difficult to produce; they are very small, most often between the size of a marble and a golf ball, and most species do not tuberize in the field, so they have to be grown in winter greenhouses or crossed with adapted cultivated forms. It would therefore be very helpful to identify predictors of high folate tubers. To this end, we are currently genotyping for single nucleotide polymorphisms (SNPs) a segregating population from a cross between a high folate $S$. boliviense individual (accession PI 597736) with a diploid S. tuberosum clone. We are also examining the possibility of correlation between leaf, seed and tuber folate content and other tuber characteristics, such as $\mathrm{pH}$, which all could decrease the time and effort needed to screen a large number of individuals.

Once identified, high folate individuals should be used to introgress the high folate trait(s) into $S$. tuberosum tetraploid cultivars adapted for commercial production. The species evaluated in this study have different ploidy levels $(2 \times, 4 \times$ or $6 \times)$ (Table 1$)$ and belong to different crossability groups. S. tuberosum subsp. andigenum is cultivated like $S$. tuberosum cultivars and very easy to introgress. We have obtained hybrids from a cross between a high folate individual from the accession PI 225710 and a diploid $S$. tuberosum clone. These hybrids were grown in the field and are currently being evaluated for folate. Both $S$. boliviense and $S$. vernei, which had high folate individuals, should be very easy to move into the cultivar genepool by $2 \mathrm{n}$ gametes or by making $4 \times$ versions of the wild species.

If the high folate trait(s) are successfully introgressed into modern potatoes, such as Russet Burbank, new commercial potato cultivars could contain double the amount of folate compared to currently-grown cultivars. Based on the current per capita consumption of $50 \mathrm{~kg}$ per year, or $137 \mathrm{~g}$ per day in the United States, such a potato would provide around $11 \%$ of the recommended daily need of $400 \mu \mathrm{g}$, assuming $20 \%$ dry matter and $80 \%$ retention during cooking. The highest folate concentrations measured in this study (e.g., $>2000 \mathrm{ng} / \mathrm{g}$ dry weight or $>400 \mathrm{ng} / \mathrm{g}$ fresh weight, assuming $20 \%$ dry matter) were higher than those found in lettuce, snap beans and oranges ( 300 to $380 \mathrm{ng} / \mathrm{g}$ fresh weight, according to the USDA Nutrient Database), for instance, but still much lower than high folate sources, such as beans, lentils and spinach ( 2000 to $6000 \mathrm{ng} / \mathrm{g}$ fresh weight). The increase obtained by genetic means could be further increased by optimizing the time of harvest, as young tubers contain up to two-fold the amount found in mature tubers [27], the storage conditions, as folates accumulate up to two-fold in tubers stored at cold temperature [21], and the cooking method, as studies show the retention rate fluctuating between $50 \%$ and $110 \%$ depending on the cooking method and the cultivar [28]. The reported folate retention rate for processed potatoes (i.e., French fries) is usually high ( $>75 \%)$ compared to boiled potatoes, for instance. This may be due to the shorter cooking time and the insolubility of folates in cooking oil during processing. In addition, potatoes destined for processing 
are often stored for several months at a cold temperature. With the increasing consumption of processed potatoes in the United States, introgression into a processing cultivar may have the most impact on the U.S. population's folate intake.

In the future, screening of additional individuals within promising accessions and species should help determine whether screening should focus on these specific accessions and species. Future research should also investigate the stability of the high folate genotypes across environments and the heritability of the high folate trait(s). Finally, the development of fast and easy-to-use predictors for high folate, such as molecular markers, is essential to accelerate the screening of potato genotypes and to assess the full potential of the potato genetic diversity.

\section{Conclusions}

In conclusion, this study highlights the genetic potential of potato for folate biofortification and illustrates the importance of collecting and evaluating exotic germplasm for improving potato varieties.

\section{Acknowledgments}

We thank Matthew Warman, Mark Barnett and Solomon Yilma for technical help. Bruce Robinson was supported by a National Needs Graduate Student Fellowship from the USDA National Institute of Food and Agriculture and a Fellowship from the USDA Western Sustainable Agriculture Research and Education.

\section{Appendix}

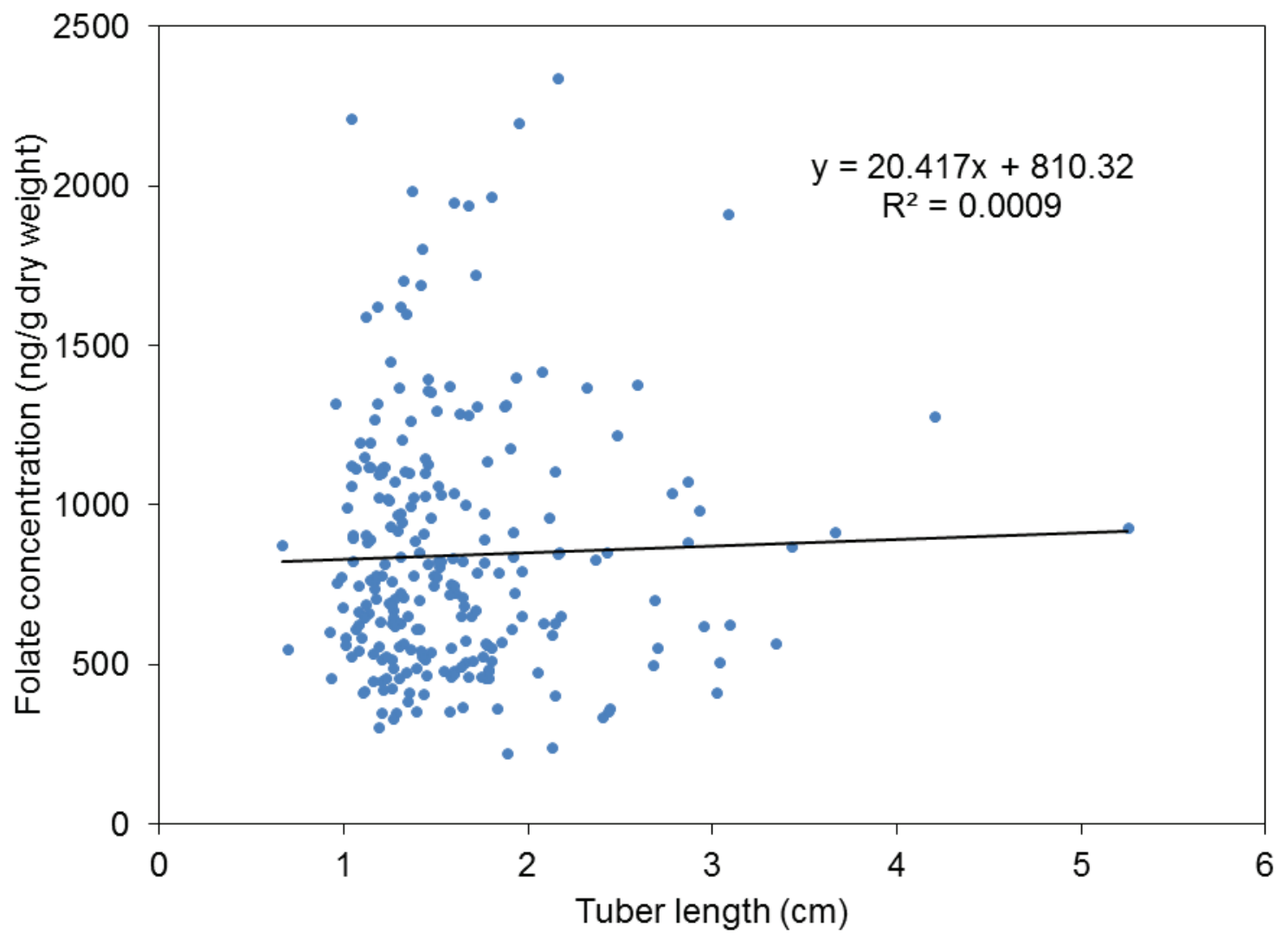

Figure A1. Relationship between folate concentration and tuber length. 


\section{Author Contributions}

Bruce R. Robinson and Aymeric Goyer conceived of and designed the experiments. Bruce R. Robinson performed the experiments. Bruce R. Robinson and Aymeric Goyer analyzed the data. Vidyasagar Sathuvalli and John Bamberg helped conceive of the study and provided critical review of the manuscript. John Bamberg provided potato seeds. Bruce R. Robinson and Aymeric Goyer wrote the paper.

\section{Conflicts of Interest}

The authors declare no conflict of interest.

\section{References}

1. Bekaert, S.; Storozhenko, S.; Mehrshahi, P.; Bennett, M.J.; Lambert, W.; Gregory, J.F., 3rd; Schubert, K.; Hugenholtz, J.; van Der Straeten, D.; Hanson, A.D. Folate biofortification in food plants. Trends Plant Sci. 2008, 13, 28-35.

2. Appling, D.R. Compartmentation of folate-mediated one-carbon metabolism in eukaryotes. FASEB J. 1991, 5, 2645-2651.

3. Hyde, J.E. Exploring the folate pathway in plasmodium falciparum. Acta Trop. 2005, 94, 191-206.

4. Shane, B.; Stokstad, E.L.R. Vitamin B12-folate interrelationships. Annu. Rev. Nutr. 1985, 5, 115-141.

5. Bailey, L.B.; Rampersaud, G.C.; Kauwell, G.P.A. Folic acid supplements and fortification affect the risk for neural tube defects, vascular disease and cancer: Evolving science. J. Nutr. 2003, 133, 1961S-1968S.

6. Beaudin, A.E.; Stover, P.J. Folate-mediated one-carbon metabolism and neural tube defects: Balancing genome synthesis and gene expression. Birth Defects Res. C Embryo Today 2007, 81, 183-203.

7. Bazzano, L.A.; He, J.; Ogden, L.G.; Loria, C.; Vupputuri, S.; Myers, L.; Whelton, P.K.; Kasner, S.E. Dietary intake of folate and risk of stroke in US men and women. Stroke 2002, 33, 1183-1189.

8. Roberts, S.H.; Bedson, E.; Hughes, D.; Lloyd, K.; Moat, S.; Pirmohamed, M.; Slegg, G.; Tranter, R.; Whitaker, R.; Wilkinson, C.; et al. Folate augmentation of treatment-Evaluation for depression (folated): Protocol of a randomised controlled trial. BMC Psychiatry 2007, doi:10.1186/1471-244X-7-65.

9. Coppen, A.; Bolander-Gouaille, C. Treatment of depression: Time to consider folic acid and vitamin B12. J. PsychopharmacoL. 2005, 19, 50-65.

10. Araújoa, J.R.; Martelb, F.; Borgesc, N.; Araújod, J.M.; Keating, E. Folates and aging: Role in mild cognitive impairment, dementia and depression. Ageing Res. Rev. 2015, 22, 9-19.

11. Storozhenko, S.; Ravanel, S.; Zhang, G.-F.; Rébeillé, F.; Lambert, W.; van der Straeten, D. Folate enhancement in staple crops by metabolic engineering. Trends Food Sci. Technol. 2005, 16, 271-281.

12. Konings, E.J.M.; Roomans, H.H.S.; Dorant, E.; Goldbohm, R.A.; Saris, W.H.M.; van den Brandt, P.A. Folate intake of the dutch population according to newly established liquid chromatography data for foods. Am. J. Clin. Nutr. 2001, 765-776.

13. Bentley, T.G.K.; Willett, W.C.; Weinstein, M.C.; Kuntz, K.M. Population-level changes in folate intake by age, gender, and race/ethnicity after folic acid fortification. Am. J. Public Health 2006, 96, 2040-2047. 
14. Blancquaert, D.; de Steur, H.; Gellynck, X.; van der Straeten, D. Present and future of folate biofortification of crop plants. J. Exp. Bot. 2014, 65, 895-906.

15. CIP. Annual Report 2014. Cross-Cutting Efforts Optimize Food Security, Nutrition and Livelihood. International Potato Center: Lima, Peru, 2015; Volume 2015.

16. Committee, P.C.G. Potato Vulnerability Statement 2014; USDA Agricultural Research Service Germplasm Resources Information Network, 2014. Available online: http://www.ars-grin.gov/ (accessed on 2 March 2015).

17. Millam, S. Agrobacterium-mediated transformation of potato. In Transgenic Crops of the World: Essential Protocols; Kluwer Academic Publishers: Dordrecht, The Netherlands, 2004; pp. 257-270.

18. Alfthan, G.; Laurinen, M.S.; Valsta, L.M.; Pastinen, T.; Aro, A. Folate intake, plasma folate and homocysteine status in a random Finnish population. Eur. J. Clin. Nutr. 2003, 57, 81-88.

19. Vahteristo, L.; Lehikoinen, K.; Ollilainen, V.; Varo, P. Application of an hplc assay for the determination of folate derivatives in some vegetables, fruits and berries consumed in finland. Food Chem. 1997, 59, 589-591.

20. Hatzis, C.M.; Bertsias, G.K.; Linardakis, M.; Scott, J.M.; Kafatos, A.G. Dietary and other lifestyle correlates of serum folate concentrations in a healthy adult population in Crete, Greece: A cross-sectional study. Nutr. J. 2006, doi:10.1186/1475-2891-5-5.

21. Goyer, A.; Navarre, D.A. Determination of folate concentrations in diverse potato germplasm using a trienzyme extraction and a microbiological assay. J. Agric. Food Chem. 2007, 55, 3523-3528.

22. Goyer, A.; Sweek, K. Genetic diversity of thiamin and folate in primitive cultivated and wild potato (solanum) species. J. Agric. Food Chem. 2011, 59, 13072-13080.

23. Pfeiffer, C.M.; Rogers, L.M.; Gregory, J.F.I. Determination of folate in cereal-grain food products using trienzyme extraction and combined affinity and reversed-phase liquid chromatography. J. Agric. Food Chem. 1997, 45, 407-413.

24. Spooner, D.M.; Ghislain, M.; Simon, R.; Jansky, S.H.; Gavrilenko, T. Systematics, diversity, genetics, and evolution of wild and cultivated potatoes. Bot. Rev. 2014, 80, 283-383.

25. Horne, D.W.; Patterson, D. Lactobacillus casei microbiological assay of folic acid derivativesin 96-well microtiter plates. Clin. Chem. 1988, 34, 2357-2359.

26. Van Daele, J.; Blancquaert, D.; Kiekens, F.; van Der Straeten, D.; Lambert, W.E.; Stove, C.P. Folate profiling in potato (Solanum tuberosum) tubers by ultrahigh-performance liquid chromatography-tandem mass spectrometry. J. Agric. Food Chem. 2014, 62, 3092-3100.

27. Goyer, A.; Navarre, D.A. Folate is higher in developmentally younger potato tubers. J. Sci. Food Agric. 2009, 89, 579-583.

28. Navarre, D.A.; Goyer, A.; Shakya, R. Nutritional value of potatoes: Vitamin, phytonutrient and mineral content. In Advances in Potato Chemistry and Technology; Singh, J., Lovedeep Kaur, L., Eds.; Elsevier Inc.: Philadelphia, United States, 2009; pp. 395-424.

(C) 2015 by the authors; licensee MDPI, Basel, Switzerland. This article is an open access article distributed under the terms and conditions of the Creative Commons Attribution license (http://creativecommons.org/licenses/by/4.0/). 